\title{
Energy Impact of Outdoor Small Cell Backhaul in Green Heterogeneous Networks
}

\author{
Agapi Mesodiakaki ${ }^{\star}$, Ferran Adelantado ${ }^{\dagger}$, Angelos Antonopoulos ${ }^{\star, \diamond}$, Elli Kartsakli ${ }^{\star}$, Luis Alonso ${ }^{\star}$, Christos Verikoukis ${ }^{\diamond}$ \\ ${ }^{\star}$ Technical University of Catalonia (UPC), Signal Theory and Communications Department, Castelldefels, Spain \\ $\dagger$ Open University of Catalonia (UOC), Barcelona, Spain \\ ${ }^{\diamond}$ Telecommunications Technological Centre of Catalonia (CTTC), Castelldefels, Spain \\ Email: \{agapi.mesodiakaki, ellik, luisg\}@tsc.upc.edu, ferranadelantado@uoc.edu, \{aantonopoulos, cveri\}@cttc.es
}

\begin{abstract}
Small cells (SCs) are expected to be densely deployed during the next few years to enhance the network capacity of future heterogeneous networks (HetNets). Due to their dense deployment, not all SCs are expected to have a direct connection to the core network. As a result, some SCs will forward their traffic to the neighboring SCs until they reach the core network, thus forming a multi-hop backhaul (BH) network. Due to the large number of $\mathrm{BH}$ links, the $\mathrm{BH}$ is expected to be one of the main challenges that future HetNets will have to face. At the same time, traffic demands are growing exponentially resulting in higher energy consumption. Therefore, how to achieve high network energy efficiency becomes of utmost importance. To that end, in this paper, we study the role of $\mathrm{BH}$ in future outdoor HetNets aiming to answer to whether or not it could constitute an energy bottleneck for the HetNet. To gain insights, we study the BH energy consumption impact compared to the access network under different traffic distribution scenarios and $\mathrm{BH}$ technologies.
\end{abstract}

Index Terms-Energy efficiency, green communications, LTEAdvanced, multi-hop backhaul, outdoor small cells.

\section{INTRODUCTION}

The overall mobile data traffic is expected to grow to 15.9 exabytes per month by 2018 , nearly an 11 -fold increase over 2013 [1]. Thus, it becomes urgent for mobile operators to maintain capacity growth to meet these new demands. At the same time, serving more traffic results in higher energy consumption. Therefore, how to achieve high network energy efficiency becomes of utmost importance [2], [3].

To that end, small cells (SCs) constitute a promising solution [4]. In particular, when SCs are deployed: i) the distance between user equipments (UEs) and base stations $(\mathrm{BSs})^{1}$ is reduced and thus the signal-to-interference-plusnoise ratio (SINR) increases and ii) the area spectral efficiency (bps $/ \mathrm{Hz} / \mathrm{m}^{2}$ ) increases (i.e., for a given bandwidth, more UEs can be served in a specific area). Therefore, dense SC deployment is expected during the next few years, with neighboring SCs being eventually as close as $50 \mathrm{~m}$ apart [4].

In such architectures, not all SCs will have a direct connection to the core network. Thus, [5]-[7] consider the introduction of optional aggregation gateways between the SCs and the core network to provide better scalability to the network by forming a multi-hop backhaul $(\mathrm{BH})$. Assuming that the

${ }^{1}$ In this paper, we will use the term BS to refer to an eNB and/or a SC. operator already has a radio network in place, a straightforward option is to consider a macro-launched topology, i.e., to connect the SC directly to the eNB site, especially in cases where fiber access is available [6]-[8]. Then, between SCs, chain, tree or mesh $\mathrm{BH}$ topologies can be used to provide further connectivity [7]. In such multi-hop BH topologies, due to the large number of $\mathrm{BH}$ links, the $\mathrm{BH}$ network dimensioning becomes more complicated, the capital expenditure increases and so does the network energy consumption. Therefore, although most works in the literature only consider the access network (AN) [9], [10], future heterogeneous network (HetNet) architecture calls for backhaul-aware strategies.

To that end, in [11], [12], Tombaz et al. stress the need for jointly considering the $\mathrm{BH}$ and $\mathrm{AN}$ energy consumption when studying the energy efficiency of future HetNets. In [11], the impact of indoor femtocell deployment on the energy efficiency of wireless networks is investigated, taking into account the $\mathrm{BH}$ power consumption. In [12], the $\mathrm{BH}$ impact on the total network power consumption is studied under different $\mathrm{BH}$ architectures and capacity requirements. It is shown that $\mathrm{BH}$ can potentially become an issue in dense SC HetNets, since it can amount to up to $50 \%$ of the total network power consumption. However, the $\mathrm{BH}$ architecture scenarios and technologies of the aforementioned works concerned exclusively indoor scenarios. Nevertheless, further study is needed for outdoor scenarios, since the outdoor environmental characteristics impose new challenges (e.g., dependency on weather conditions, larger distances, many interference sources), and thus different types of $\mathrm{BH}$ solutions may be required (e.g., home digital subscriber (DSL) lines are not available).

Thus, in this paper, we study the $\mathrm{BH}$ energy impact in outdoor scenarios where several SCs backhaul their traffic to the neighboring SCs until they reach the core network. We study the relation between $\mathrm{AN}$ and $\mathrm{BH}$ power consumption and we shed light to whether or not $\mathrm{BH}$ could become an energy bottleneck for the network. Finally, to gain valuable insights on the topic, we provide extensive simulation results under different traffic distribution scenarios and $\mathrm{BH}$ technologies.

The rest of the paper is organized as follows: In Section II the system model is described. In Sections III and IV, the energy efficiency analysis together with the calculation of the main involved parameters and the most popular wireless $\mathrm{BH}$ 


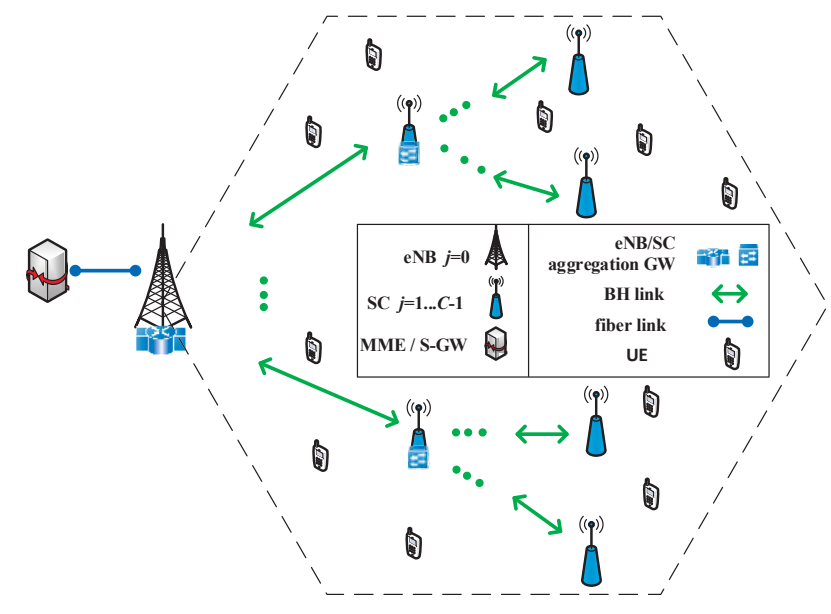

Fig. 1. System model.

solutions for outdoor SC HetNets are presented, respectively. In Section V, simulation results and useful insights on the topic are provided, while Section VI concludes the paper.

\section{SySTEM MODEL}

We consider an LTE-A eNB sector ${ }^{2}$, which consists of a set of BSs $\mathcal{C}=\{0,1, \ldots, j, \ldots, C-1\}$ : one eNB $(j=0)$ overlaid with $C-1$ SCs $(j=1, \ldots, C-1)$, as depicted in Fig. 1 , where $C$ is the cardinality of the set $\mathcal{C}$. We focus on the downlink and make the following assumptions:

- Each UE is associated with the BS that provides the highest received signal. In particular, the user association is based on the reference signal received power (RSRP), which measures the average received power over the resource elements that carry cell-specific reference signals within certain frequency bandwidth [5].

- Each SC is connected to the core network through the eNB aggregation gateway either directly or through one or more $\mathrm{SC}$ aggregation gateways.

- We consider a set $\mathcal{L}=\left\{\mathcal{L}_{1}, \mathcal{L}_{2}, \ldots, \mathcal{L}_{k}, \ldots, \mathcal{L}_{K}\right\}$ of outof-band $\mathrm{BH}$ links and a fiber link from the eNB site to the core network, as depicted in Fig. 1. Each BH link $k$ is represented by a set $\mathcal{L}_{k}$ that includes all cells $j$ that backhaul their traffic through it.

- We assume that the total transmit power of each BS is equally distributed among its subcarriers.

- There is a set of $\mathcal{N}=\{1, \ldots, i, \ldots, N\}$ fixed UEs with strict guaranteed bit rate (GBR) QoS requirements, $r_{i}$ based on their service/application.

- There is a maximum number of physical resource blocks (PRBs) available to each $\mathrm{BS} j$, denoted by $N_{P R B_{j, \max }}$.

\section{ENERGY EFFICIENCY ANALYSIS}

For reader's convenience, we provide in this section the calculation of the most important parameters that affect the total network energy efficiency. In particular, the SINR calculation

\footnotetext{
${ }^{2} \mathrm{We}$ assume that inter-sector interference is mitigated through some form of fractional frequency reuse scheme or sophisticated frequency allocation [14], thus focusing our analysis on a single eNB sector.
}

is given in Section III-A, while the power consumption models for both the AN and $\mathrm{BH}$ are presented in Section III-B.

\section{A. SINR calculation}

The SNR received by UE $i$ from $\mathrm{BS} j$ is given by [15]

$$
\begin{array}{r}
S N R_{i j(d B)}=P_{j s u b(d B m)}+G_{T_{x j}(d B i)}-L_{c b_{j}(d B)} \\
-L_{p_{i j}(d B)}-L_{f_{i j}(d B)}-N_{t h(d B m)}-N F_{(d B)}
\end{array}
$$

where $P_{j s u b}=10 \log _{10}\left(P_{j_{\max }} /\left(12 N_{j} N_{P R B_{j, \max }}\right)\right)$ is the power allocated by $\mathrm{BS} j$ to each subcarrier, with $P_{j_{\max }}$ denoting its maximum transmission power in $\mathrm{mW}, N_{j}$ the number of antennas of BS $j$ and $N_{P R B_{j, \max }}$ the maximum number of PRBs allocated to it (please note that $1 \mathrm{PRB}$ is equal to 12 subcarriers in the frequency domain and $0.5 \mathrm{~ms}$ in the time domain). The parameter $G_{T_{x j}}$ represents the antenna gain and $L_{c b_{j}}$ the cable loss between the radio RF connector and the antenna, while $L_{p_{i j}}$ the pathloss between UE $i$ and BS $j$ and $L_{f_{i j}}$ the losses due to slow-fading. Then, $N_{t h}$ refers to the thermal noise and $N F$ to the noise figure. Hence, the SINR received by user $i$ from BS $j$ can be expressed as

$$
S I N R_{i j(d B)}=S N R_{i j(d B)}-10 \log _{10}\left(\frac{I}{N}+1\right)
$$

where $I$ is the total interference (in watts) experienced by UE $i$ from the interfering BSs in the eNB sector, which depends on the applied frequency allocation scheme. Finally, $N$ denotes the total noise power (in watts) received by $\mathrm{UE} i$.

\section{B. Power consumption models}

1) Access link: The power consumed in the AN link between a BS $j$ and a UE $i$ can be calculated as

$$
P_{A N_{i j}}=\frac{P_{j_{\max }}}{N_{j} N_{P R B_{j_{\max }}}}\left\lceil\frac{r_{i}}{f\left(S I N R_{i j}\right)}\right\rceil
$$

where the first fraction denotes the power allocated to a PRB pair by BS $j$ (equal to one subframe time $(1 \mathrm{~ms}$ ) in the time domain) and the second represents the number of PRB pairs needed for the association of UE $i$ with the BS $j$, where $[\cdot]$ is the ceiling function operator. The parameter $r_{i}$ denotes the throughput demand of UE $i$ and $f\left(S I N R_{i j}\right)=B_{P R B} \log _{2}\left(1+S I N R_{i j}\right)$ is its spectral efficiency, with $B_{P R B}$ denoting the bandwidth of a PRB pair.

2) Backhaul link: The power consumed in a $\mathrm{BH}$ link $k$, represented by the $\mathrm{BH}$ link set $\mathcal{L}_{k}$, can be given by

$$
P_{B H_{\mathcal{L}_{k}}}=g\left(\sum_{i \in \mathcal{N}} \sum_{j \in \mathcal{L}_{k}} r_{i} x_{i j}\right)
$$

where $x_{i j}$ is the association vector that is equal to 1 when UE $i$ is associated with $\mathrm{BS} j$ and 0 otherwise and $g(\cdot)$ is a function that maps the aggregated throughput that passes through the link (i.e., the sum of the total throughput of all the SCs that backhaul their traffic through the link $\mathcal{L}_{k}$, namely $\forall j \in \mathcal{L}_{k}$ ) to a specific power consumption. Specifically, given that adaptive modulation and coding is used, the aggregated throughput that passes through the link is mapped to a target SINR (inverse mapping compared to $f(\cdot)$ ) and then the power consumption of the link is calculated from the link budget equation similarly to (2) depending on the technology used for the $\mathrm{BH}$. 


\section{Energy efficiency}

The network energy efficiency can be expressed as the total number of successfully transmitted bits by all UEs divided by the total energy consumption (i.e., the sum of the energy consumed in the AN and BH links). Equivalently, it can be expressed as the total throughput of all UEs divided by the total power consumption and thus can be formulated as

$$
E E=\frac{\sum_{i \in \mathcal{N}} \sum_{j \in \mathcal{C}} r_{i} x_{i j}}{\sum_{i \in \mathcal{N}} \sum_{j \in \mathcal{C}} P_{A N_{i j}} x_{i j}+\sum_{\mathcal{L}_{k} \in \mathcal{L}} P_{B H_{\mathcal{L}_{k}}}}
$$

\section{WIRELESS BACKHAUL SOLUTIONS}

In this section, we analyze the different $\mathrm{BH}$ solutions that can be employed for backhauling future outdoor HetNets. We will focus our study on wireless $\mathrm{BH}$ solutions, since wired solutions are more difficult to implement under the considered scenarios (e.g., although fiber can provide very high capacity, its deployment is very costly and can take many years). In particular, the following main $\mathrm{BH}$ solutions are considered: millimitre wave, microwave, and sub- $6 \mathrm{GHz}$ band [4].

\section{A. Millimetre wave (mmWave)}

Millimetre wave (mmWave) $(60,70-80 \mathrm{GHz}$, also known as the V- and E-band, respectively) [16] is expected to be one of the main $\mathrm{BH}$ solutions especially for scenarios as the ones previously described with multiple hops. Due to the high path loss at these frequencies, high gain antennas with narrow beams are required. Therefore, mmWave frequencies enable only short range point-to-point (PTP) line-of-sight (LOS) radio links and the connection to the local aggregation gateway would most probably require a number of hops. On the other hand, the high path loss and narrow beams reduce the risk of interfering with other mmWave radio links.

At the same time, the huge amount of available bandwidth at high frequencies can provide significant capacity enhancement. Lower frequency technologies rely on complex RF techniques to deliver higher capacities, such as multipath propagation and channel aggregation below $6 \mathrm{GHz}$, or spatial multiplexing at higher frequencies up to $42 \mathrm{GHz}$. In contrast, mmWave technologies rely on generous availability of wideband RF-channels to deliver Gbps of throughput using simple single-channel configurations, which gives mmWave a potential for cost-per-bit advantage for high capacity BH [17].

\section{B. Microwave}

Microwave (6-60 GHz) is widely used, mostly because of the low deployment cost. Microwave technologies support link throughputs of multiple Mbps. Specifically, the link capacity $(C)$ is given by the following formula

$$
C_{(M b p s)}=B_{B H(M H z)} S E_{(b p s / H z)}
$$

where $B_{B H}$ stands for the channel bandwidth and $S E$ for the spectral efficiency. Microwave channel sizes are typically multiples of $7 \mathrm{MHz}$, while modern-day microwave equipment can transmit over multiple adjacent channels of up to $56 \mathrm{MHz}$.

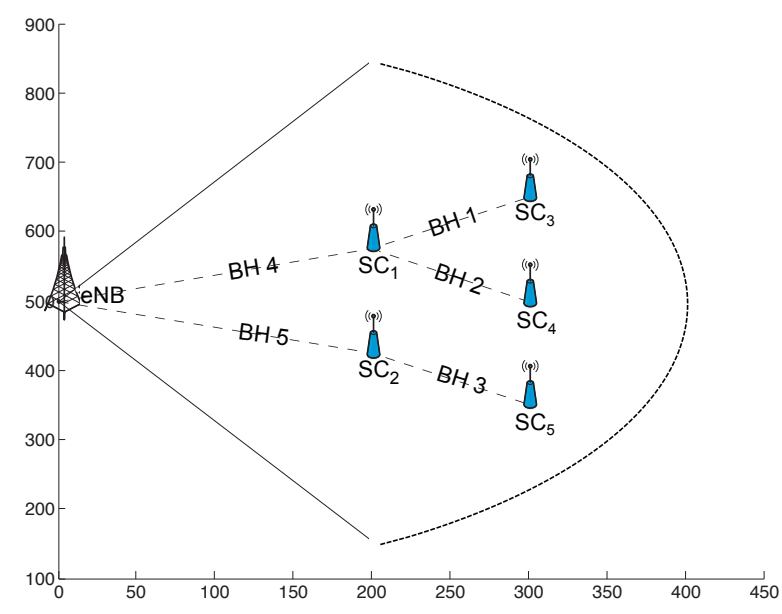

Fig. 2. Simulation scenario.

With modern transmission methods, the capacity of a microwave radio link can be boosted up to $1 \mathrm{Gbps}$ [4]. Depending on the frequency and link budget, microwave frequencies allow a coverage range from a few hundreds of meters to a few kilometers. Similarly with mmWave frequencies, the microwave frequencies require narrow beams to cope with the path loss. However, the beams are wider than mmWave beams, requiring less accurate antenna alignment. While the mmWave frequencies are suitable for densely placed base stations, microwave frequencies are more suitable for long distance rooftop-to-rooftop connections due to less available spectrum and lower path loss.

\section{Sub-6GHz band}

Sub-6GHz spectrum is able to operate in harsh mobile nonline-of-sight (NLOS) propagation environments. Therefore, it is considered suitable as a $\mathrm{BH}$ solution for small cells deployed in locations where high capacity NLOS connectivity is required. The available spectrum at this band is generally ranging from 5 to $20 \mathrm{MHz}$, while sub- $6 \mathrm{GHz}$ frequencies can achieve NLOS coverage of some kilometers.

\section{Performance Evaluation}

\section{A. Scenarios}

In our extensive simulations in MATLAB ${ }^{\circledR}$, we considered an eNB sector, which overlaps $5 \mathrm{SCs}$, as depicted in Fig 2. The employed BH technologies were: i) LOS mmWave links $\left(f_{B H 1}=60 \mathrm{GHz}\right.$ band $)$ of $B_{B H 1}=100 \mathrm{MHz}$ channel bandwidth [16], ii) LOS microwave links $\left(f_{B H 2}=28 \mathrm{GHz}\right)$ of $B_{B H 2}=28 \mathrm{MHz}$ [18], and iii) sub- $6 \mathrm{GHz}\left(f_{B H 3}=3 \mathrm{GHz}\right)$ of $B_{B H 3}=10 \mathrm{MHz}$ [19]. We assume that the channels allocated to the eNB and to the SCs are orthogonal, while SCs that are adequately far from each other may reuse the same bands.

In each realization (1000 in total), we consider $N$ UEs of different throughput requirements. Specifically, $10 \%$ of UEs demand $512 \mathrm{kbps}, 10 \% 728 \mathrm{kbps}$ and 80\% $1024 \mathrm{kbps}$. We further consider two UE traffic distribution scenarios. In the first, the UEs are uniformly distributed in the sector area of radius $R$, while in the second they form hotspots. In scenario $2,2 / 3$ of the total traffic is generated in a radius $r=70 \mathrm{~m}$ 
TABLE I

Simulation VALUES

\begin{tabular}{|c|c|c|c|}
\hline Parameter & Value & Parameter & Value \\
\hline$f_{A N}$ & $2.0 \mathrm{GHz}$ & $\overline{B_{e N B}, B_{S C}}$ & $10 \mathrm{MHz}$ \\
\hline$N_{P R B_{S C \max }}, N_{P R B_{e N B \max }}$ & 50 & $N F$ & $9 \mathrm{~dB}$ \\
\hline$P_{S C_{\max }}, P_{e N B_{\max }}$ & $30,46 \mathrm{dBm}$ & $N_{t h}$ & $-174 \mathrm{dBm} / \mathrm{Hz}$ \\
\hline$L_{p_{e N B}}$ & $69.55+26.16 \log f_{A N}-13.82 \log h_{e N B}-C_{H}+\left(44.9-6.55 \log h_{e N B}\right) \log d, d$ in $\mathrm{km}$ & $G_{T_{x S C}}, G_{T_{x e N B}}$ & $5,14 \mathrm{dBi}$ \\
\hline$L_{p_{S C}}$ & $69.55+26.16 \log f_{A N}-13.82 \log h_{S C}+\left(44.9-6.55 \log h_{S C}\right) \log d, d$ in $\mathrm{km}$ & $R$ & $450 \mathrm{~m}$ \\
\hline$C_{H}$ & $0.8+\left(1.1 \log f_{A N^{-}}-0.7\right) h_{m}-1.56 \log f_{A N}$ & $r$ & $70 \mathrm{~m}$ \\
\hline$h_{m}, h_{S C}, h_{e N B}$ & $1.5,2.5,25 \mathrm{~m}$ & $N_{e N B}, N_{S C}$ & 2 \\
\hline
\end{tabular}

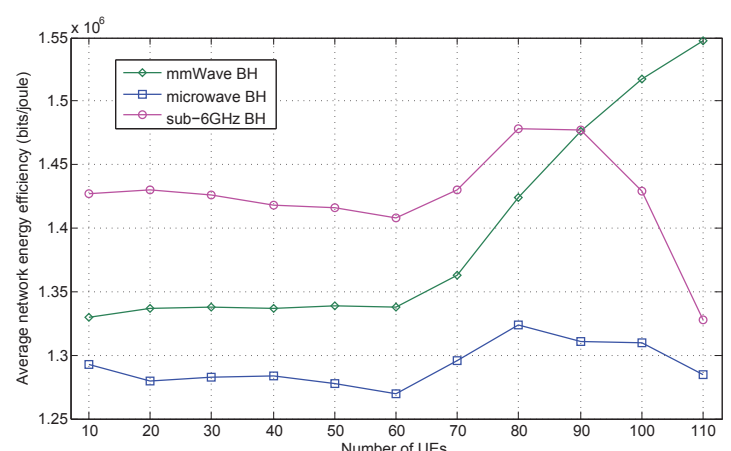

(a) Uniform traffic.

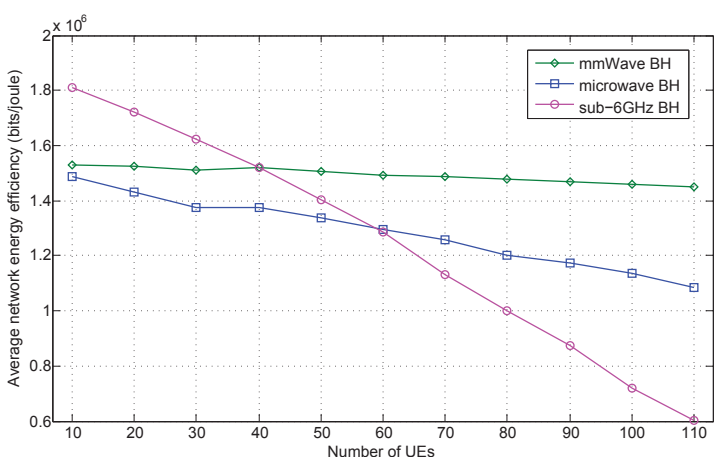

(b) Hotspot traffic.

Fig. 3. Average energy efficiency under different BH technologies for (a) uniform and (b) hotspot traffic.

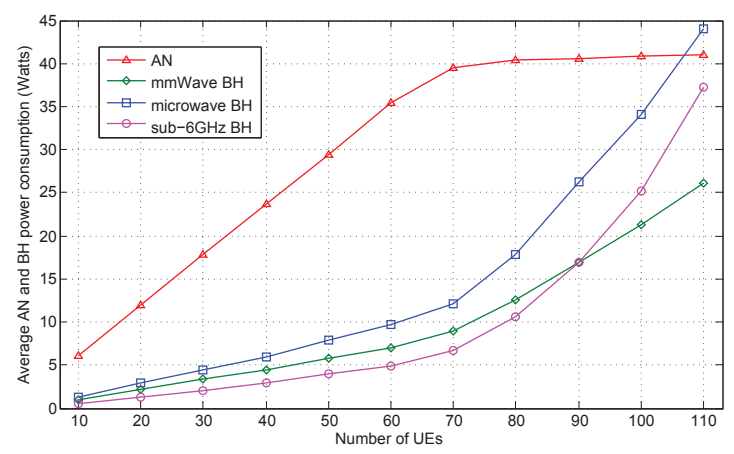

(a) Uniform traffic.

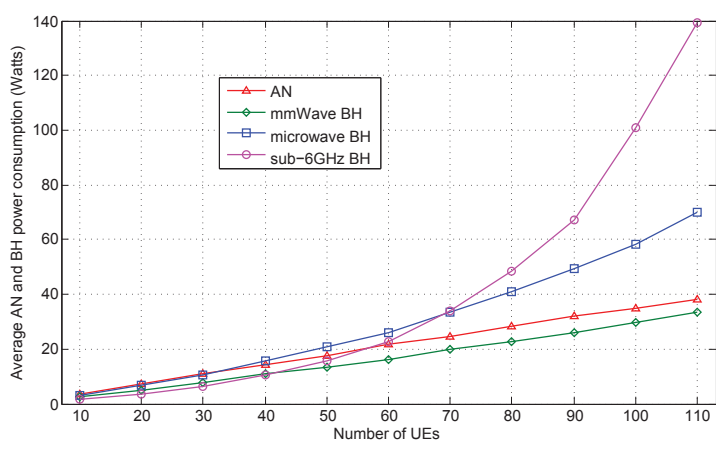

(b) Hotspot traffic.

Fig. 4. Average AN and BH power consumption under different BH technologies for (a) uniform and (b) hotspot traffic.

from SC3 and SC4 according to 3GPP [20] and the rest is uniformly distributed in the sector area. Notice that scenario 2 is more realistic, as in future HetNets, UEs are expected to be very close to SCs and to generate bursty hotspot traffic [4].

All simulation parameters are summarized in Table I, where $f_{A N}$ denotes the frequency used in the AN. Moreover, $h_{e N B}$ and $h_{S C}$ denote the BS antenna height of the eNB $(j=0)$ and the SCs $(j \neq 0)$, respectively. Furthermore, $h_{m}$ denotes the mobile antenna height, while $C_{H}$ stands for the antenna height correction factor and $d$ for the distance between the BS and the UE. The slow fading is modeled by a log-normal random variable with zero mean and deviation 8 or $10 \mathrm{~dB}$ in the case the signal is transmitted by an eNB or a SC, respectively.

\section{B. Results}

The range of the parameter $N$ has been appropriately selected to avoid system overloading, and thus all UE throughput demands are satisfied (i.e., the UE QoS is guaranteed) in all experiments. Therefore, for a given number of UEs, the differences among the total network energy efficiency of each $\mathrm{BH}$ technology only depend on the BH energy consumption.

To that end, in Fig. 3(a) and 3(b) the average network energy efficiency is depicted for different $\mathrm{BH}$ technologies under uniform and hotspot traffic, respectively. As it can be observed, for uniform traffic and low number of UEs (i.e., low traffic), the sub- $6 \mathrm{GHz} \mathrm{BH}$ solution presents the highest energy efficiency. This stems from the fact that this technology experiences lower path loss compared to the others and thus for the same traffic it requires less energy consumption (see 4(a)). However, as $N$ increases, the $\mathrm{BH}$ traffic increases and consequently the available bandwidth of each BH technology becomes very important. Due to the high bandwidth availability of $60 \mathrm{GHz}, \mathrm{mmWave}$ shows the best performance for very high traffic, since it is able to send high amount of data without increasing the transmitted power. On the contrary, the available bandwidth of sub- $6 \mathrm{GHz}$ is very limited. Therefore, for high traffic (i.e., 80-90 UEs), a significant increase in the transmitted power of the $\mathrm{BH}$ links is required, as depicted in 


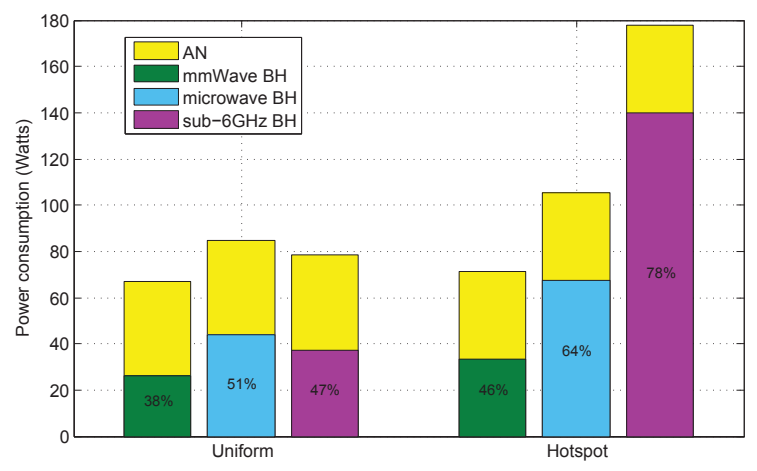

Fig. 5. AN and $\mathrm{BH}$ power consumption under different $\mathrm{BH}$ technologies for high uniform and hotspot UE traffic $(N=110)$.

4(b), which results in lower energy efficiency.

To gain further insights to whether or not $\mathrm{BH}$ could constitute an energy bottleneck, we depict in Fig. 4(a) and 4(b) the $\mathrm{BH}$ power consumption compared to the AN for every $\mathrm{BH}$ technology for uniform and hotspot traffic, respectively. As it is shown, the $\mathrm{AN}$ is much more significant than the $\mathrm{BH}$ power consumption when the UEs are uniformly distributed in the sector area. This is due to the fact that i) most UEs in this case receive stronger signal from the eNB and thus get connected to it and ii) the AN power consumption in the case a UE is connected to the eNB is much higher than when connected to a SC. Also notice that the AN power consumption increases at a high rate as the number of UEs increases, since more UEs get connected to the eNB. However, for very high traffic there are no resources left in the eNB and thus more UEs get connected to SCs, which results in a smoother increase in the AN power consumption. On the other hand, in the case of hotspot traffic more UEs get connected to SCs and thus the $\mathrm{BH}$ power consumption becomes higher than the AN.

The $\mathrm{BH}$ power consumption constitutes a significant part, which becomes more important as the number of UEs increases. For very high traffic $(N=110)$, as depicted in Fig. 5, $\mathrm{BH}$ power consumption can reach up to $38 \%, 51 \%$ and $47 \%$ of the total power consumption for uniform traffic and $46 \%, 64 \%$ and $78 \%$ of the total power consumption for hotspot traffic, when mmWave, microwave and sub-6GHz are employed, respectively. Therefore, we conclude that future HetNets call for backhaul-aware strategies. In addition, mmWave seems the best choice to avoid a potential energy bottleneck mainly due to its very high bandwidth availability. However, given that it requires LOS, a mixture of the studied $\mathrm{BH}$ technologies is anticipated to be used in future HetNets.

\section{CONCLUSION}

In this paper, we studied the $\mathrm{BH}$ energy impact in future green HetNets under different UE traffic scenarios and $\mathrm{BH}$ technologies. It was shown that the $\mathrm{BH}$ constitutes a significant part of the total power consumption, which becomes more important as the number of UEs (i.e., total traffic) increases. The BH energy impact was also shown to be dependent on the UE traffic distribution. Specifically, for hotspot traffic the BH energy impact becomes more significant, since more UEs get connected to SCs (i.e., the BH traffic increases). At the same time, the fact that future HetNets are expected to deal with very high amount of hotspot traffic, predicates the need for backhaul-aware algorithm design. Finally, it was shown that mmWave seems the best choice to avoid a potential energy bottleneck mainly due to its very high bandwidth availability. However, given that it requires LOS, a mixture of the studied $\mathrm{BH}$ technologies is anticipated for future SC HetNets.

\section{ACKNOWLEDGMENT}

This work has been funded by AGAUR (2014-SGR-1551) and by the research projects GREENET (PITN-GA-2010264759) and GEOCOM (TEC2011-27723-C02-01).

\section{REFERENCES}

[1] “Cisco Visual Networking Index: Global Mobile Data Traffic Forecast Update, 2013-2018," Cisco, Feb. 2014.

[2] N. Chilamkurti, S. Zeadally, and F. Mentiplay, "Green Networking for Major Components of Information Communication Technology Systems," EURASIP J. Wireless Commun. and Netw., vol. 2009, no.1, pp. 656-785, Dec. 2009.

[3] A. P. Bianzino, C. Chaudet, D. Rossi, and J.-L. Rougier, "A survey of Green Networking research," IEEE Comm. Surveys \& Tutorials, vol. 14, no.1, pp. 3-20, Feb. 2012.

[4] "Backhaul Technologies for Small Cells: Use Cases, Requirements and Solutions," Small Cell Forum, Rel. 1, 049.01.01, Feb. 2013.

[5] EUTRA \& EUTRAN Overall Description, 3GPP TS 36.300, v. 11.5.0, Rel. 11, Mar. 2013.

[6] "Small Cell Backhaul Requirements," NGMN Alliance, v. 1.0, Jun. 2012.

[7] "Small cells address the growing demand for data," Tellabs, Sep. 2012.

[8] "Release Three: Urban Foundations," Small Cell Forum, Rel. 3, 103.03.01, Feb. 2014.

[9] Y. S. Soh, T. Q. S. Quek, M. Kountouris, and H. Shin, "Energy efficient heterogeneous cellular networks," IEEE J. Sel. Areas Commun., vol. 31, no. 5, pp. 840850, May 2013.

[10] A. Mesodiakaki, F. Adelantado, L. Alonso, and C. Verikoukis, "Energyefficient User Association in Cognitive Heterogeneous Networks," IEEE Commun. Mag., Energy-Efficient Cognitive Radio Networks, vol. 52, no. 7, pp. 22-29, Jul. 2014.

[11] S. Tombaz, Z. Zheng, and J. Zander, "Energy efficiency assessment of wireless access networks utilizing indoor base stations," in Proc. IEEE PIMRC, Sep. 2013, pp. 3105-3110.

[12] S. Tombaz et al., "Is Backhaul Becoming a Bottleneck for Green Wireless Access Networks?," in Proc. IEEE ICC, Jun. 2014, pp. 4029-4035.

[13] ABI Research, "Outdoor small cell market to reach $\$ 1.8 B$ this year," http://www.abiresearch.com/press/more-outdoor-small-cellswill-ship-in-2012-than-ma

[14] D. Lopez-Perez, C. Xiaoli, and I. Guvenc, "On the expanded region of picocells in heterogeneous networks," IEEE J. Sel. Topics Signal Process., vol. 6, no. 3, pp. 281-294, Jun. 2012.

[15] H. Holma and A. Toskala, Eds., LTE for UMTS: OFDMA and SC-FDMA based radio access, John Wiley \& Sons, Mar. 2009.

[16] K.-C. Huang and Z. Wang, Millimeter Wave Communication Systems, Wiley, Mar. 2011.

[17] A. Ghosh et al., "Millimeter-Wave Enhanced Local Area Systems: A High-Data-Rate Approach for Future Wireless Networks," IEEE J. Sel. Areas Commun., vol. 32, no. 6, pp. 1152-1163, Jun. 2014.

[18] Y. Azar et al., "28 GHz Propagation Measurements for Outdoor Cellular Communications Using Steerable Beam Antennas in New York City,' in Proc. IEEE ICC, Jun. 2013, pp. 1550-3607.

[19] V.S. Abhayawardhana et al., "Comparison of Empirical Propagation Path Loss Models for Fixed Wireless Access Systems," in Proc. IEEE VTC, May 2005, pp. 73-77.

[20] Small cell enhancements for E-UTRA and E-UTRAN-Physical layer aspects, 3GPP TS 36.872, v. 1.0.0, Rel. 12, Aug. 2013 